\title{
MICROMACHINED POLYCRYSTALLINE DIAMOND HEMISPHERICAL SHELL RESONATORS
}

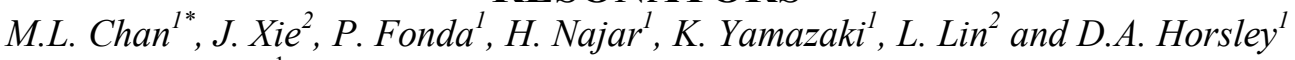 \\ ${ }^{1}$ University of California Davis, California, USA \\ ${ }^{2}$ University of California Berkeley, California, USA
}

\begin{abstract}
We present the development of millimeter scale 3D hemispherical shell resonators fabricated from polycrystalline diamond, a material with low thermoelastic damping and very high stiffness. Resonators are fabricated through a combination of electro discharge machining (EDM) and silicon micromachining techniques, to achieve $1 \mathrm{~mm}$ diameter 3D highly axisymmetric hemispherical shell structures. By using piezoelectric actuation and optical characterization, the elliptical wineglass vibration mode is determined to be at $34.86 \mathrm{kHz}$, with the two degenerate wineglass modes having a relative frequency mismatch of $2 \%$.
\end{abstract}

\section{INTRODUCTION}

The commercial hemispherical resonator gyro (HRG) is a high-precision and highly reliable solid state gyroscope that meets the inertial grade performance [1]. The topology of an axisymmetric shell mounted on a stem minimizes unwanted coupling to the base substrate and, along with the use of highpurity fused-quartz materials, results in high $\mathrm{Q}$ operation. By operating these structures in a rate integrating mode, the sensor can continue to integrate the applied rotation during power interruptions allowing for robust long-term operation.

These characteristics have spurred recent interest in the development of microscale HRGs that can be mass manufactured in a wafer scale level to achieve low-cost inertial grade sensors with a small volume and large dynamic range. To achieve modematching and high-Q performance in a hemispherical resonator, geometric uniformity and symmetry in combination with low thermoelastic damping structural material are critical. Many different methods such as wafer scale glass blowing [2], traditional silicon etching $[3,4]$ and integrating precision machining with standard silicon microfabrication [5,6] have been employed to create $3 \mathrm{D}$ spherical or hemispherical shells. Materials such as polysilicon, and glass have also been used as the isotropic structural material for the shell itself.

In this work, we describe the development and characterization of millimeter scale 3D hemispherical shell resonators. Micro-electro discharge machining is first employed to fabricate the silicon mold from which the shell structure can be built. Using traditional silicon micromachining techniques to carry out the subsequent polish, deposition and release steps, we realized a $1 \mathrm{~mm}$ diameter 3D shell resonator. Polycrystalline diamond is used as the structural material due to its unique material properties such as high stiffness and low thermoelastic damping.

\section{FABRICATION}

In order to realize complex 3D shapes such as hemispherical wine-glass resonators, resonators are fabricated through a combination of electro discharge machining (EDM) and silicon micromachining techniques, as shown in Figure 1. By leveraging the advantages of $\mu$ EDM's precision and orientation-independent material removal-rate, highly symmetric and geometrically-precise mold features can be first formed on silicon. Subsequent process steps based on the traditional silicon micromachining technologies are used to define the structural layers, create a free standing shell structure and allowing future integration with electrical actuation and sensing components.

\section{Micro Electro Discharge Machining ( $\mu$ EDM)}

A low resistivity (0.008-0.2 $\Omega$-cm), antimony-doped, (100) silicon substrate is first deposited with a metal hard mask consisting of $50 \mathrm{~nm}$ thick Chromium and $100 \mathrm{~nm}$ thick Gold layers. The hemispherical shell mold is then formed via a two-step EDM process where a hemispherical tool is used to machine $1 \mathrm{~mm}$ diameter hemispherical wells into the silicon substrate. A subsequent isotropic etching step in HNA (Hydrofluoric/Nitric/Acetic acids) etchant chemically polishes the inside surface of the machined hemispherical mold, while the metal hard mask protects the top surface of the silicon substrate. Image analysis demonstrates that the finished shell mold exhibits high symmetry, having a radial standard deviation below $6 \mu \mathrm{m}$ for a $500 \mu \mathrm{m}$ radius shell [6] and a surface roughness of $4 \mathrm{~nm}$ measured at the inside of the silicon mold.

a)

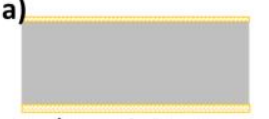

1.Au/Cr mask deposition

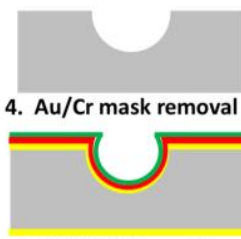

7. PE-TEOS deposition
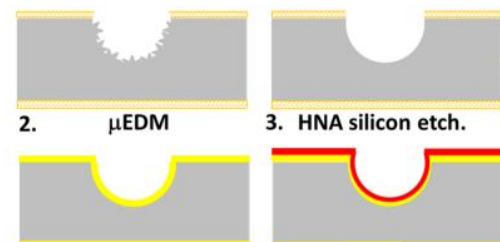

3. HNA silicon etch.
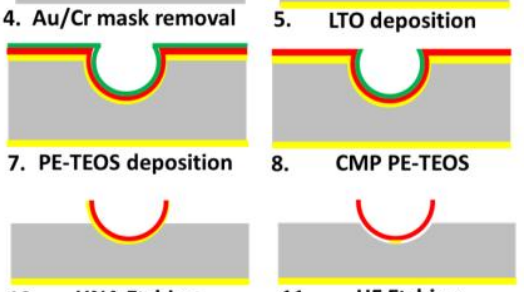

10. HNA Etching

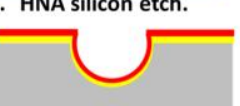

6. CVD diamond deposition

b)

11. HF Etching

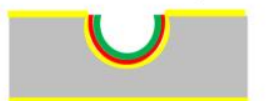

9. Diamond dry etch Legend

Silicon $\quad$ CVD Diamond LTO Oxide $\mathrm{Au} / \mathrm{Cr}$ PE-TEOS

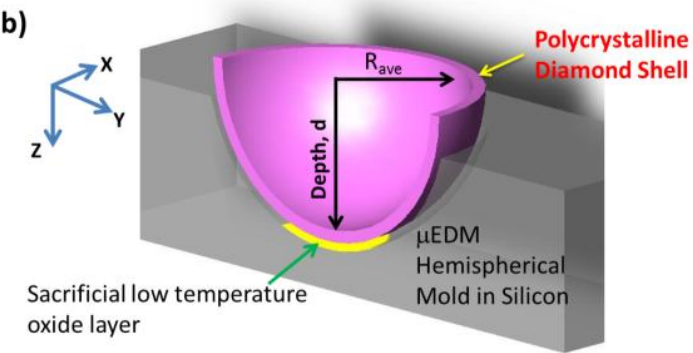

Figure 1: a) Fabrication process flow for creating CVD diamond hemispherical shell resonator, b) cross-sectional schematic of the shell structure.

\section{Silicon Micromachining}

After stripping the etch mask, a $2 \mu \mathrm{m}$ thick LTO $\mathrm{SiO}_{2}$ sacrificial layer is grown conformally on the shell mold. The shell mold is then seeded using ultrasonic seeding suspensions containing nanocrystalline diamond powders with diameter from 5-50 nm . Using methane $\left(\mathrm{CH}_{4}\right)$ concentration of $1.5 \%$ and relative boron concentration of $444 \mathrm{ppm} / \mathrm{CH}_{4}$, a $1 \mu \mathrm{m}$ thick Boron-doped diamond structural layer is deposited via hot-filament chemical vapor deposition (HFCVD, SP3 Diamond Technologies). A $5 \mu \mathrm{m}$ thick plasma enhanced tetraethyl-orthosilicate (PE-TEOS) oxide 
layer is deposited to mask the topside of the wafer. PE-TEOS is selected to ensure conformal oxide mask coverage on the rough microcrystalline diamond surface.

A chemical mechanical polishing (CMP) step (Strausbaugh) removes the oxide mask at a rate of $\sim 3000 \mathrm{~A} / \mathrm{min}$ from the wafer surface but retains the mask within the mold. A subsequent diamond plasma etching (SPTS, APS etcher) is carried out to define the shell. The diamond shells are released in 49\% HF and the top surface of the silicon mold is etched back in HNA to facilitate non-contact optical characterization of the shell structure. SEM micrographs in Fig. 2 show both a totally released diamond shell structure and an array of $1 \mathrm{~mm}$ diameter diamond hemispherical shell that has been etched back to a depth, $\mathrm{d} \sim 370 \mu \mathrm{m}$.

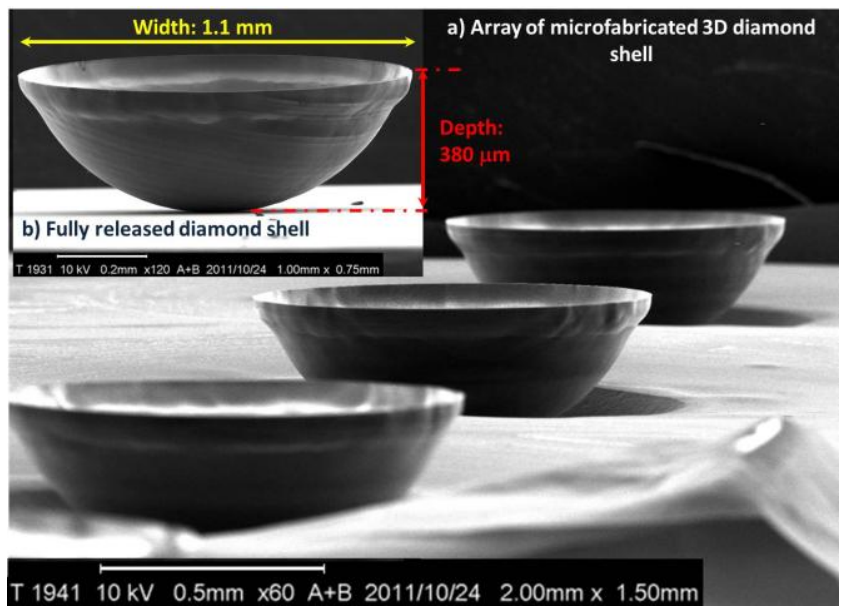

Figure 2: SEM micrographs of the diamond shell, a) shows an array of etched back diamond shells attached to the silicon substrate at the bottom of the well, (b) shows a fully released $1 \mathrm{~mm}$ diameter, 380 um deep shell.

\section{POLYCRSYTALLINE DIAMOND}

Thin-film polycrystalline diamond offers enhanced mechanical properties, including significantly higher stiffness, strength, hardness, thermal conductivity, and chemical robustness, versus silicon and most other thin-film materials commonly used in microfabrication technologies. The excellent strength to density ratio and diamond's superior surface properties make this an ideal material for low surface losses and ultra-low thermoelastic damping, both significant properties required for a resonator.

In this work, the diamond film deposited during the HFCVD process is microcrystalline diamond (MCD). Through control of the deposition conditions, either micro- or nano- crystalline diamond (NCD) films can be deposited. The selection of MCD as the structural material is motivated by the higher sp3 content of the MCD films and reduced density of grain boundaries; both translate into the high Q-factors of 71,400 at $299.86 \mathrm{MHz}$ for a diamond disk resonator [7] and 201,000 at $264 \mathrm{kHz}$ for a diamond double ended tuning fork [8].

The Raman spectra obtained on the Boron doped MCD film is shown in Fig. 3 using an excitation wavelength at $514.5 \mathrm{~nm}$. The spectrum features three different peaks: a) first order Silicon Raman peak at $520 \mathrm{~cm}^{-1}$, b) peaks at $\sim 1230$ and c) $1324.4 \mathrm{~cm}^{-1}$ (Full-width half-maximum, FWHM $=7.0 \mathrm{~cm}^{-1}$ ). The characteristic sp3 single crystal diamond is dominated by the first order Raman line at $1332 \mathrm{~cm}^{-1}$. In our measurement the peak is down shifted to $1324.4 \mathrm{~cm}^{-1}$, consistent with the previous studies on residual stress and effects of boron doping in MCD films [9]. The other peak at
$1230 \mathrm{~cm}^{-1}$ is also broadly consistent with the peaks in the phonon density of states (PDOS) of diamond and attributed to the incorporation of boron in the diamond lattice [10]. The comparison between the diamond and non-diamond components (at 1500-1550 $\left.\mathrm{cm}^{-1}\right)$, ratio of $I(1332) / I(1500)$ shows the purity of the diamond film used in the hemispherical resonator structure.

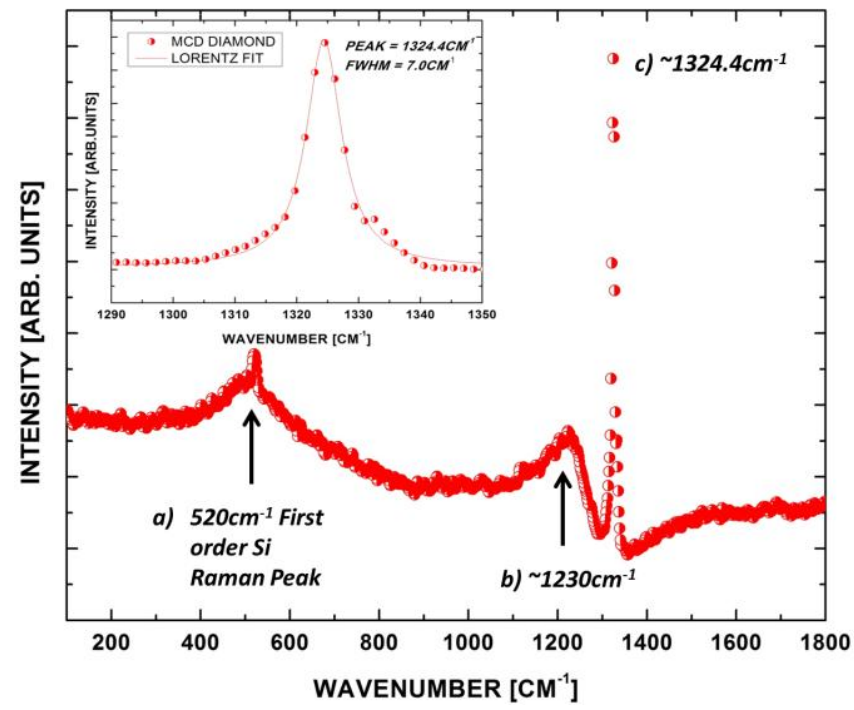

Figure 3: Raman spectra of the Boron doped microcrystalline diamond. Figure inset shows the Lorentzian fit for the first order diamond Raman peak.
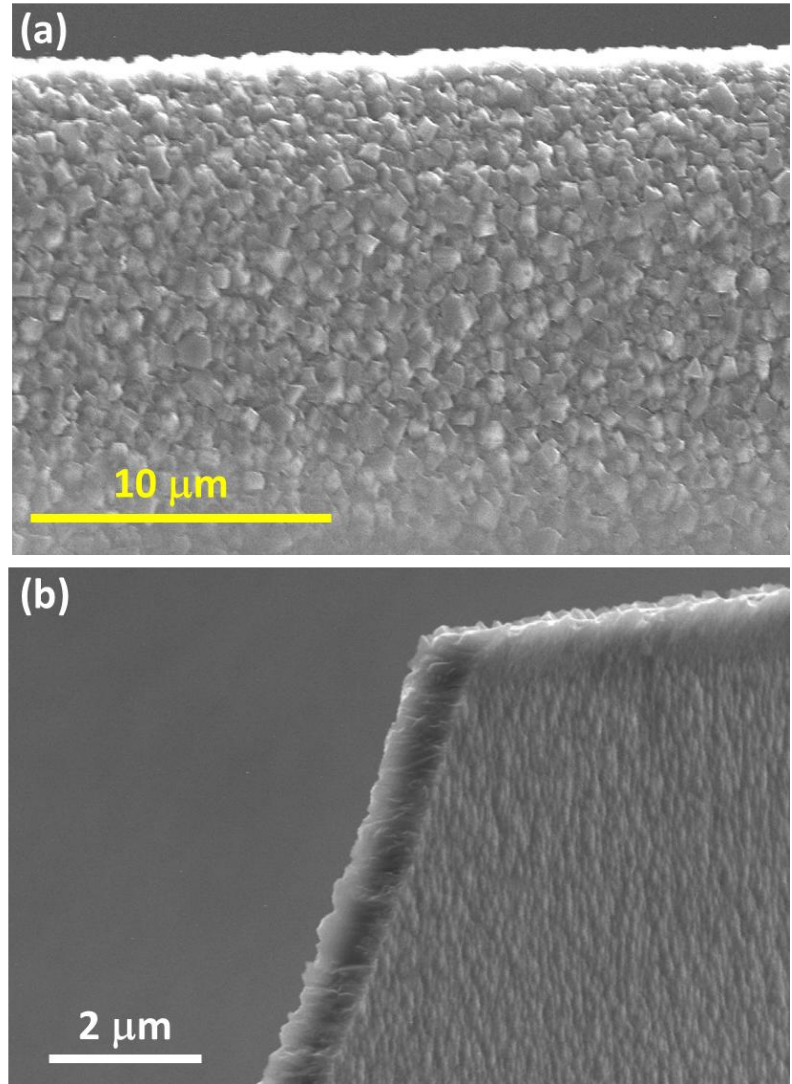

Figure 4: SEM Micrographs of a) top view of the MCD film and b) a representative sample diamond shell with a cracked rim to show the columnar nature of the MCD structural layer. 
The SEM view of the MCD shell resonator shown in Figure 4a illustrates the highly faceted surface with grain size in the range between $0.2-0.5 \mu \mathrm{m}$. Hence, the roughness of shell structure is dominated by the roughness of the MCD film layer. This roughness will increase with increasing film thickness. Fig. 4b shows part of a cracked shell to illustrate the columnar nature of the diamond growth profile from the diamond seeded layer at the base of the silicon mold. The thickness of the diamond shell is between $0.8-1.0 \mu \mathrm{m}$.

\section{TESTING AND CHARACTERIZATION}

Preliminary prototypes of the diamond hemispherical shell are characterized using a laser Doppler vibrometer (LDV) (Polytec) both in air and in vacuum. Fig. 5 shows the schematic of the testing setup, where the diamond shell was mounted onto a shear mode piezoelectric actuator (Noliac A/S) with a bandwidth of 1.7 $\mathrm{MHz}$ to excite the shell. The single point LDV laser spot is focused through a 10x objective on the rim of the shell to directly measure the displacement of the vibrating shell. A miniature rotation stage and two axis goniometer (OptoSigma) are used to tune the incidence angle of the laser on the shell for maximum signal to noise ratio. The whole setup was also mounted in a vacuum chamber (Janis) to allow measurement at sub-mTorr pressures.

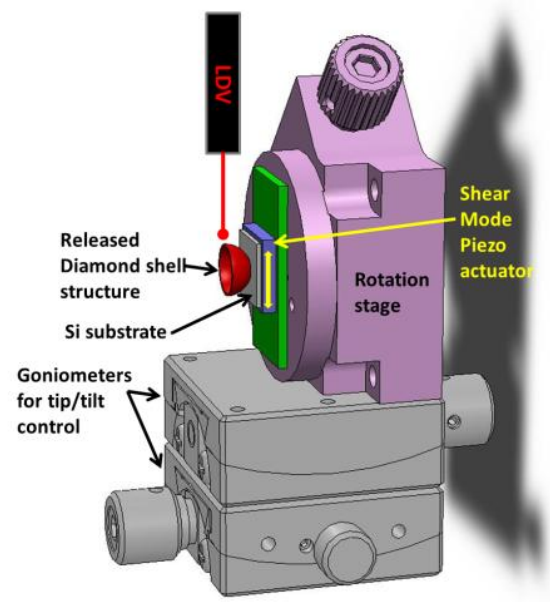

Figure 5: Schematic of experimental setup used to characterize the diamond shell.

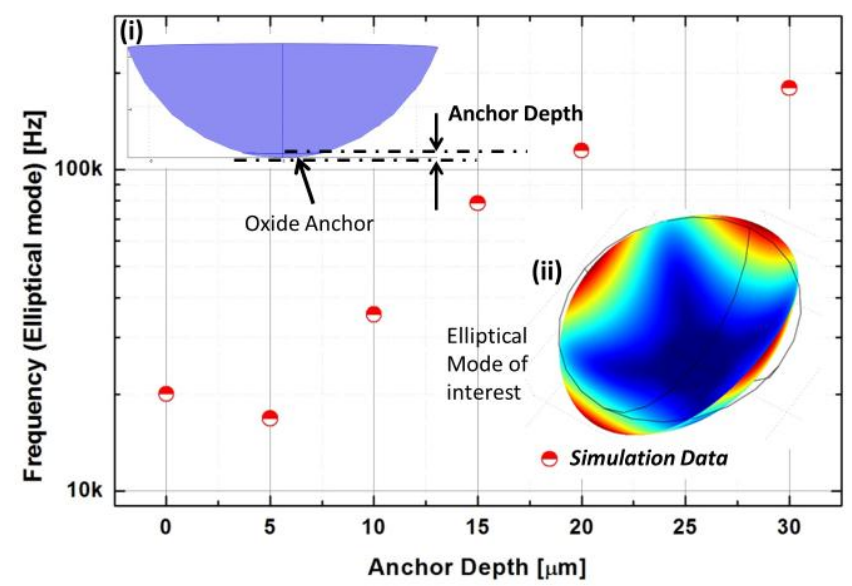

Figure 6: Finite element simulation of the elliptical mode at varying anchor depth. Inset figure: i) illustrates the definition of anchor depth and ii) shows the vibration profile at the elliptical mode.

\section{RESULTS}

\section{Finite Element Simulation}

Finite element modal analysis was carried out using COMSOL $^{\mathrm{TM}}$ to analyze the mode shapes of the hemispherical shell, and compared with the experimental results. The focus of the simulation is on the wineglass/elliptical mode, where the shell shape changes from circular to elliptical and the degenerate modes are spaced $45^{\circ}$ apart from one another. Fig. 6 plots the simulated eigenfrequency at the elliptical mode with varying anchor depth ranging from 5-30 $\mu \mathrm{m}$ and shows a dependence on the anchor depth.

\section{Frequency response}

The frequency response of the hemispherical shell in air and in vacuum at $\mathrm{P} \sim 30 \mu$ Torr are shown in Fig. $7 \mathrm{a}$ and $\mathrm{b}$. The resonance peaks at $f_{1}=34.68 \mathrm{kHz}$ and $f_{2}=35.55 \mathrm{kHz}$ correspond to the two degenerate elliptical modes, where the frequency mismatch $\Delta f / f$ is measured to be $\sim 2 \%$. Lorentzian fit to the response in vacuum extracts a Q-factor of $\sim 3000$ (Fig. $7 \mathrm{c}$ and d).
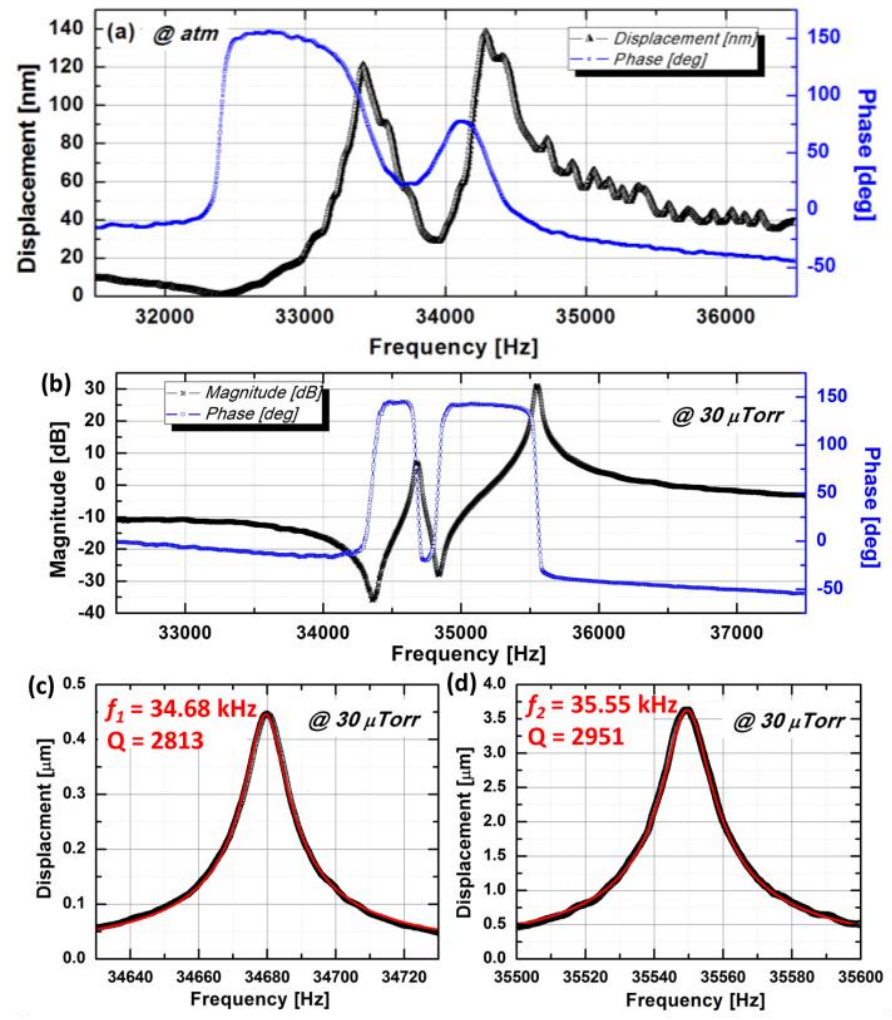

Figure 7: Measured frequency response in (a) air and (b) vacuum. Close-ups at $f_{1}$ and $f_{2}$ are shown in (c) and (d).

In order to verify the actual anchor depth, SEM of the sample is carried out, as shown in Fig. 8. The side view image shows the hemispherical shell profile after the HNA etch back, and the anchor depth is estimated to be $\sim 9 \mu \mathrm{m}$. Hence, the experimental results agree well with the simulation data with an elliptical mode frequency variation of $\sim 5 \%$. The low Q-factor and relatively large frequency mismatch can be attributed to the anchor design and the rim roughness as shown in the SEM image. The anchor is currently controlled by the time etching of silicon during the final HNA and HF etching step and the rim roughness an artifact of the initial HNA polishing etch after EDM. Both issues can be remedied with process optimization. 


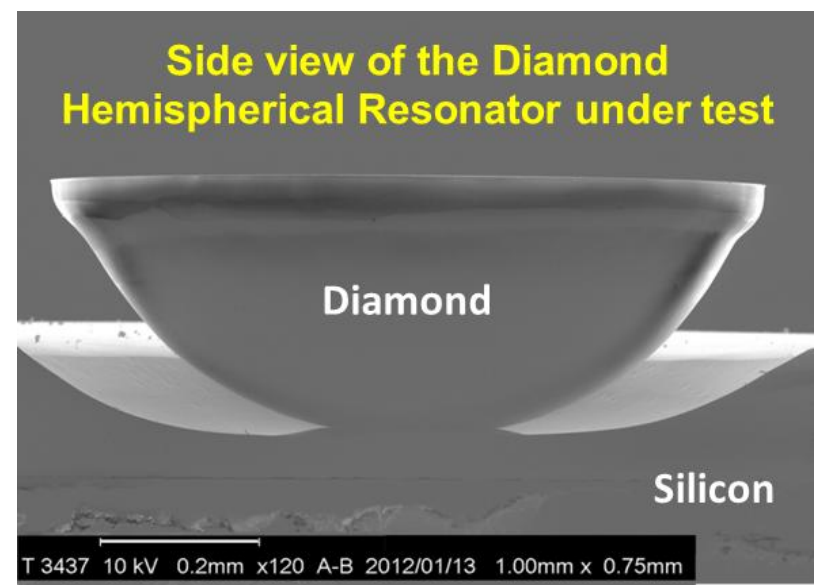

Figure 8: SEM Micrograph showing the side view of the hemispherical shell structure after the HNA floor etch.

\section{Mode Shapes}

The elliptical mode shapes at frequencies $f_{1}$ and $f_{2}$ were identified by measuring the radial vibration around the perimeter of the shell, as the shell is rotated through $360^{\circ}$. At every $10^{\circ}$ interval the frequency response of the structure is measured and the displacement at $f_{1}$ and $f_{2}$ is extracted. Fig. 9 shows the polar plot of the shell displacement as it is excited at each frequency. Each measured mode shape exhibits the 4 nodes which are characteristic of the elliptical modes. By employing a fitting function described by $\left|A_{i} \sin \left(2 \theta+\varnothing_{i}\right)\right|$, the angle between the principal axes of elasticity is extracted to be $\sim 45^{\circ}$ in good agreement with theory.

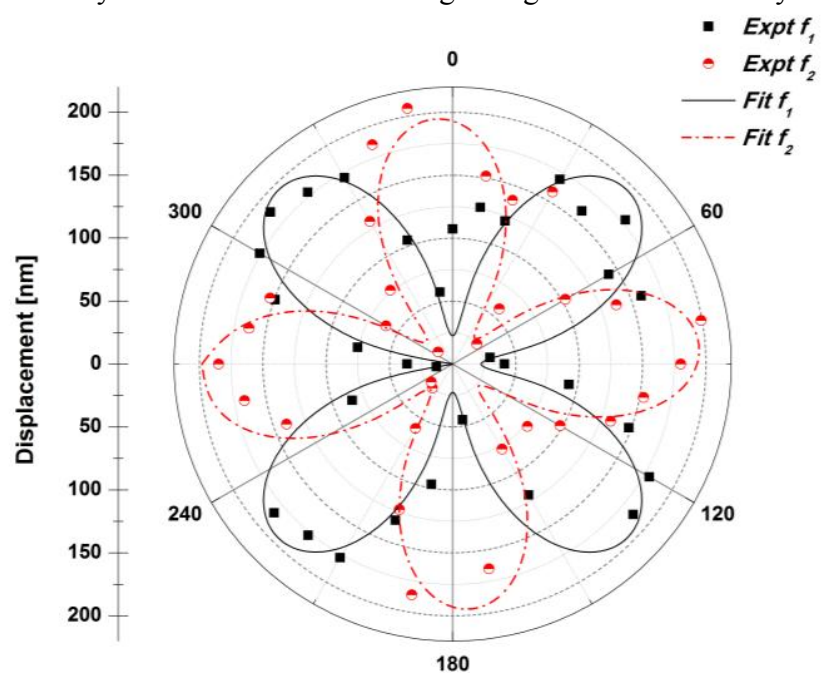

Figure 9: Measured displacement amplitude along the rim of the resonator shell with excitation at $f_{1}(\mathbf{\square})$ and $f_{2}(\theta)$ illustrating the expected elliptical mode shapes.

\section{CONCLUSION}

We developed a 3D hemispherical shaped microcrystalline diamond shell structures, and demonstrated the shells as mechanical resonant structures. Using a combination of $\mu \mathrm{EDM}$ and silicon machining technologies, 3D axisymmetric shell silicon structures can be employed as the molds from which high Q-factor material such as microcrystalline diamond can be deposited. The shells are characterized through piezoelectric excitation at the base and optical detection at the rim of the shell to determine the natural frequencies and mode shapes of the shell structure operating at the wineglass mode. The experimental results agree with the simulation for the anchor depth fabricated. Without any trimming or tuning of the natural frequencies, the frequency mismatch and Q-factor are determined to be $2 \%$ and 3000 at $34.68 \mathrm{kHz}$.

\section{ACKNOWLEDGEMENTS}

This work is supported by DARPA under grant W31P4Q-111-0003. The authors would like to thank the support of Gerry Chandler and Maria Peralta from SP3 Diamond Technologies; Alan Hicklin from UC Davis NEAT facility for Raman spectroscopy; the staff in UC Davis Northern California Nanotechnology Center and UC Berkeley Marvell Nanolab.

\section{REFERENCES}

[1] D.D. Lynch, "Hemispherical Resonator Gyro," R. R. Ragan, ed., Inertial Technology for the Future, IEEE Transactions on Aerospace Electronic Systems, Vol. AES-20, No. 4, pp. 414 444, (1984).

[2] I.P Prikhodko, S.A. Zotov, A.A. Trusov and A.M. Shkel, "Microscale glass-blown three dimensional spherical shell resonators", J. Microelectromech. Syst., 20-3, pp.691-701, (2011).

[3] L.C. Fegely, D.N. Hutchison, S.A. Bhave, "Isotropic etching of 111 SCS for wafer scale manufacturing of perfectly hemispherical silicon molds", 16th International Conference on Solid-State Sensors, Actuators and Microsystems (Transducers 11), Beijing, China, 6/5-9/2011.

[4] X. Gao, L. Sorenson, F. Ayazi, "3-D Micromachined hemispherical shell resonators with integrated capacitive transducers", IEEE $25^{\text {th }}$ Internal symposium on Micro Electro Mechanical Systems 2012, Paris, France, 1/29-2/2/2012, pp. 165-171.

[5] K. Visvanathan, L. Tao, Y. B. Gianchandani, "3D-soule: A fabrication process for large scale integration and micromachining of spherical structures", IEEE $24^{\text {th }}$ Internal symposium on Micro Electro Mechanical Systems 2011, Cancun, Mexico, 1/23-27/2011, pp. 45-48.

[6] M.L Chan, P. Fonda, C. Reyes, J. Xie, H. Najar, L. Lin, K. Yamazaki, D.A. Horsley, "Micromachining 3D hemispherical features in silicon via micro-EDM", IEEE $25^{\text {th }}$ Internal symposium on Micro Electro Mechanical Systems 2012, Paris, France, 1/29-2/2/2012, pp. 289-292.

[7] M. Akgul, R. Schneider, Z. Ren, G. Chandler, V. Yeh and C.T.C. Nguyen, "Hot filament CVD conductive microcrystalline diamond for high $\mathrm{Q}$, high acoustic velocity micromechanical resonators", Frequency Control and the European Frequency and Time Forum (FCS 2011), San Francisco, 5/2-5/2011, pp. 1-6.

[8] H. Najar, M.L. Chan and D.A. Horsley, "Impact of doping and microstructure on quality factor of CVD diamond micromechanical resonators", Frequency Control and the European Frequency and Time Forum (FCS 2012), Baltimore, Accepted.

[9] W.L. Wang, M.C. Polo, G. Sanchez, J. Cifre, J. Esteve, "Internal stress and strain in heavily boron-doped diamond films grown by microwave plasma and hot filament chemical vapor deposition", J. Appl. Phys. 80, pp. 1846 - 1851, (1996).

[10] M. Bernard, A. Deneuville and P. Muret, "Non-destructive determination of the boron concentration of heavily doped metallic diamond thin films from Raman Spectroscopy" Diamond Relat. Mater. 13, pp. 282-286, (2004).

\section{CONTACT}

*M.L. Chan, tel: +1-530-752-5180; cmlchan@ucdavis.edu 